\title{
Multi-Objective Optimization of Submerged Arc Welding Process
}

\author{
Saurav Datta* and Siba Sankar Mahapatra \\ Department of Mechanical Engineering, National Institute of Technology, Rourkela, Orissa-769008, India
}

Received 14 April 2009; accepted 5 September 2009
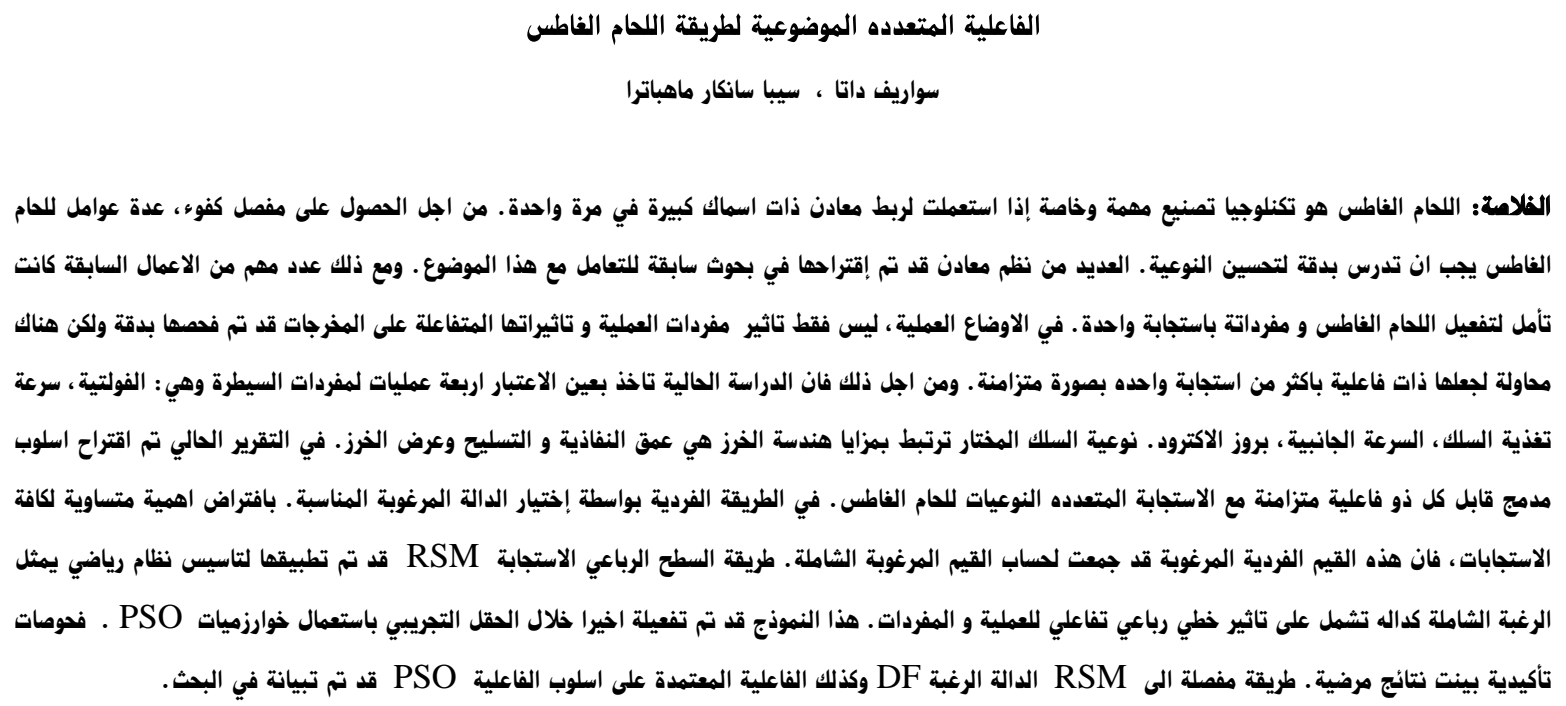

\begin{abstract}
Submerged arc welding (SAW) is an important metal fabrication technology specially applied to join metals of large thickness in a single pass. In order to obtain an efficient joint, several process parameters of SAW need to be studied and precisely selected to improve weld quality. Many methodologies were proposed in the past research to address this issue. However, a good number of past work seeks to optimize SAW process parameters with a single response only. In practical situations, not only is the influence of process parameters and their interactive effects on output responses are to be critically examined but also an attempt is to be made to optimize more than one response, simultaneously. To this end, the present study considers four process control parameters viz. voltage (OCV), wire feed rate, traverse speed and electrode stick-out. The selected weld quality characteristics related to features of bead geometry are depth of penetration, reinforcement and bead width. In the present reporting, an integrated approach capable of solving the simultaneous optimization of multi-quality responses in SAW was suggested. In the proposed approach, the responses were transformed into their individual desirability values by selecting appropriate desirability function. Assuming equal importance for all responses, these individual desirability values were aggregated to calculate the overall desirability values. Quadratic Response Surface Methodology (RSM) was applied to establish a mathematical model representing overall desirability as a function involving linear, quadratic and interaction effect of process control parameters. This model was optimized finally within the experimental domain using PSO (Particle Swarm Optimization) algorithm. A confirmatory test showed a satisfactory result. A detailed methodology of RSM, desirability function (DF) and a PSO-based optimization approach was illustrated in the paper.
\end{abstract}

Keywords: Submerged arc welding (SAW), Multi-objective optimization, Response surface methodology (RSM), Desirability function (DF), Particle swarm optimization (PSO)

\section{Introduction}

Submerged arc welding (SAW) is a multi-factor, multiobjective metal joining technology in which several process control parameters interact in a complicated manner and influence differently on quality of the prepared 
weld. Weld quality depends on various features of bead geometry, mechanical-metallurgical characteristics of the weld as well as on weld chemistry. Moreover, the cumulative effect of combined aforesaid quality features determines the extent of joint strength that determines functional aspects if the weld is subjected to practical field of application. Therefore, preparation of a satisfactory good quality weld seems to be a challenging job. Complete knowledge regarding the mode of influence of the process control parameters and their interactions are to be exactly known prior to select an optimal process environment capable of producing desired quality weld. However, SAW optimization is a difficult task due to simultaneous fulfillment of multi-quality features which should be close to the desired target value at the optimal setting. In practice, it may happen that an improvement of one response may cause severe loss to another quality feature for a particular parametric combination.

Tay and Butler (1996) proposed an application of an integrated method using experimental designs and neural network technologies for modeling and optimizing a metal inert gas (MIG) welding process. Correia et al. (2004) used Genetic Algorithm (GA) to decide near-optimal settings of a GMAW welding process. Dongcheol et al. (2002) suggested the use of Genetic Algorithm and Response Surface Methodology (RSM) for determining optimal welding conditions. Hsien-Yu Tseng (2006) proposed an integrated approach to address the welding economic design problem. The integrated approach applied general regression neural network (NN) to approximate the relationship between welding parameters (welding current, electrode force, welding time, and sheet thickness) and the failure load. An analytical formula was generated from the trained general regression neural network, and the mathematical model for the economic welding design was constructed. GA was then applied to resolve the mathematical model and to select the optimum welding parameters. These parameters were recommended for use to obtain the preferred welding quality at the least possible cost.

Zhao et al. (2006) focused on the performance -predicting problems in the spot welding of the body-galvanized steel sheets. Artificial Neural Networks (ANNs) were used to describe the mapping relationship between welding parameters and welding quality. After analyzing the limitation that existed in standard back propagation (BP) networks, the original model was optimized based on a lots of experiments. A lot of experimental data about welding parameters and corresponding spot-weld quality were provided to the ANN for study. The results showed that the improved BP model can predict the influence of welding currents on nugget diameters, weld indentation and the shear loads ratio of spot welds. The forecasting precision was quite high satisfying the practical application value. Pasandideh and Niaki (2006) presented a new methodology for solving multi-response statistical optimization problems. This methodology integrates desirability function and simulation approach with a genetic algorithm. The desirability function was used for modeling the multi-response statistical problem whereas the simulation approach generated required input data and finally the genetic algorithm was implemented to optimize the model.

Praga-Alejo et al. (2008) highlighted that the Neural Network (NN) with GA as a complement are good optimization tools. The authors compared its performance with the RSM that is generally used in the optimization of the process, particularly in welding.

Many designed experiments require the simultaneous optimization of multiple responses. The common trend to tackle such an optimization problem is to develop mathematical models of the responses. These indicate the entire process behavior. The effect of process parameters on different responses can be analyzed from the developed models. Multiple linear regression and Response Surface Methodology are two common tools available for developing the mathematical models of the responses as a function of process parameters. Depending on the requirement, each quality features/responses are optimized (maximized or minimized) to determine the optimal setting of the parameters. However, this method is applicable for the optimization of a single objective function. In a multiobjective case, it is essential to convert these multiple objectives to an equivalent single objective function which has to be optimized finally.

A common approach is to use a desirability function combined with an optimization algorithm to find the most desirable settings. In the desirability function approach, individual response desirability values are calculated depending on the target as well as prescribed tolerance limit of the response variables. Individual desirability values are then aggregated to calculate the overall desirability value. The optimal setting is one which can maximize the overall desirability. In doing so, a mathematical model is required for overall desirability. The model is then optimized finally. However, as the number of factors that affect the complexity of a multiple response problem increases, conventional optimization algorithms can fail to find the global optimum. For these situations, a common approach is to implement a heuristic search procedure like the GA and ANN or other optimization algorithms like Controlled Random Search (CRS) Price, W. L. (1977). However, it has been found that GA was adapted many times by previous researchers; less effort was made on application of CRS and even PSO in optimizing features of submerged arc weld. In consideration of the above, the present study aims at evaluating a near optimal parameter setting for the optimization of bead geometry parameters of a submerged arc weld. The study proposes integrating RSM-based desirability function approach and a PSO algorithm for multi-response optimization of SAW. Bead geometry parameters of submerged arc weld on mild steel were selected as multi-objective responses and they were optimized to select the optimal process environment. Finally, the study concludes the effectiveness and application feasibility of the proposed integrated approach. 


\section{Desirability Function (DF) Approach}

Individual desirability values related to each of the quality parameters are calculated using the formula proposed by Derringer and Suich in1980.

There are three types of desirability function: Lowerthe-Better (LB), Higher-the-Better (HB) and Nominalthe-Best (NB). In the present investigation, for reinforcement and bead width LB criteria; and for penetration depth HB criteria have been selected. This is because, the objective of the work was to minimize reinforcement and bead width (to reduce weld metal consumption) and to maximize penetration depth as strength of the welded joint directly depends on penetration depth. The NB criterion is generally selected in cases where responses have their fixed target value.

An individual desirability value using the Lower-thebetter (LB) criterion is shown in Fig. 1. The value of $\hat{y}$ is expected to be the lower the better. When $\hat{y}$ is less than a particular criteria value, a desirability value $d_{i}$ equal to 1 ; if $\hat{y}$ exceeds a particular criteria value, the desirability value equals to $0 . d_{i}$ varies within the range 0 to 1 . The desirability function of the Lower-thebetter (LB) criterion can be written as below (Eqs. 1 to 3). Here, $y_{\min }$ denotes the lower tolerance limit of $\hat{y}$, the $y_{\max }$ represents the upper tolerance limit of $\hat{y}$ and $r$ represents the desirability function index, which is to be assigned previously according to the consideration of the optimization solver. If the corresponding response is expected to be closer to the target, the index can be set to the larger value, otherwise a smaller value.

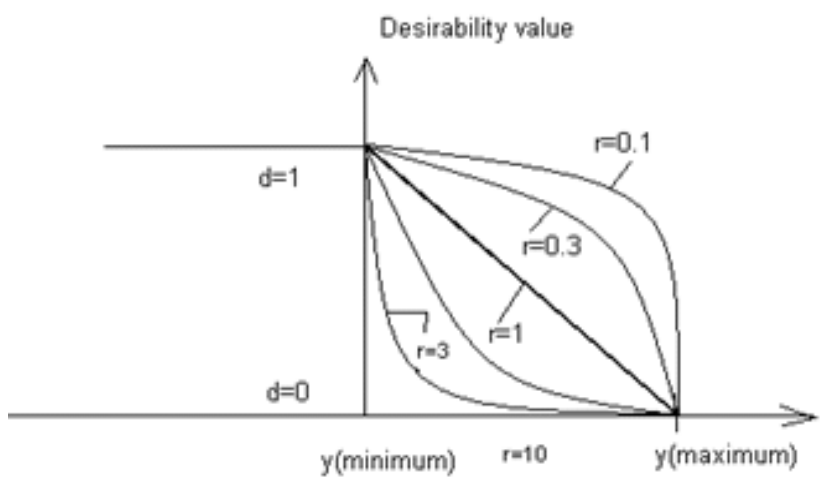

Desirability function (Lower-the-better)

Figure 1. Desirability Function (LB)

If $\hat{y} \leq y_{\min }, d_{i}=1$

If $y_{\text {min }} \leq \hat{y} \leq y_{\text {max }}, d_{i}=\left(\frac{\hat{y}-y_{\text {max }}}{y_{\text {min }}-y_{\max }}\right)^{r}$

If $\hat{y} \geq y_{\max }, d_{i}=0$
An individual desirability value using the Higherthe-better (HB) criterion is shown in Fig. 2. The value of $\hat{y}$ is expected to be the higher the better. When $\hat{y}$ is exceeds a particular criterion value, according to the requirement, the desirability value $d_{i}$ is equals to 1 ; if $\hat{y}$ is less than a particular criteria value, ie. less than the acceptable limit, the desirability value is equals to 0 . The desirability function of the Higher-the-better (HB) criterion can be written as below (Eqs. 4 to 5). Here, $y_{\text {min }}$ denotes the lower tolerance limit of $\hat{y}$, the $y_{\max }$ represents the upper tolerance limit of $\hat{y}$ and $r$ represents the desirability function index, which must have been previously according to the consideration of the optimization solver. If the corresponding response is expected to be closer to the target, the index can be set to the larger value, otherwise to a smaller value.

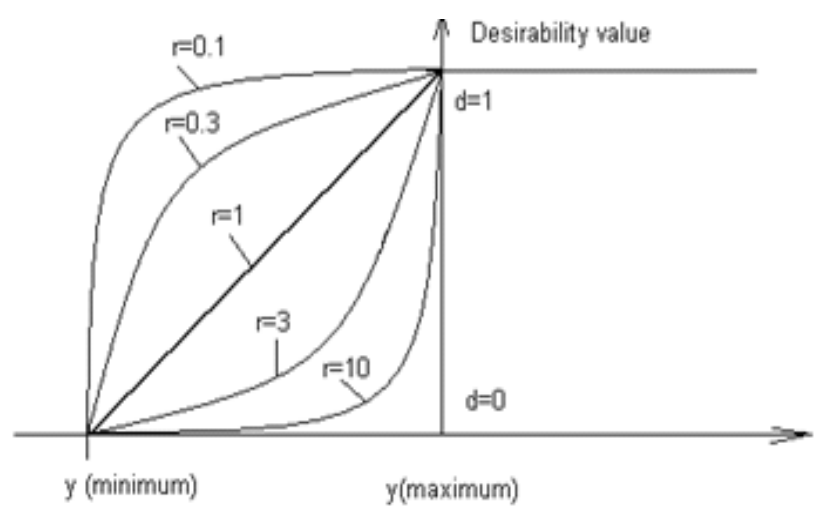

Desirability function (Higher-the-better)

Figure 2. Desirability Function (HB)

$$
\begin{aligned}
& \text { If } \hat{y} \leq y_{\text {min }}, d_{i}=0 \\
& \text { If } y_{\text {min }} \leq \hat{y} \leq y_{\text {max }}, d_{i}=\left(\frac{\hat{y}-y_{\text {min }}}{y_{\text {max }}-y_{\text {min }}}\right)^{r}
\end{aligned}
$$

If $\hat{y} \geq y_{\max }, d_{i}=1$

The individual response desirability values were accumulated to calculate the overall desirability, using the following Eq. (7). Here, $D$ is the overall desirability value, $d_{i}$ is the individual desirability value of ith quality characteristic and $n$ is the total number of responses. $w_{i}$, is the individual response weightage.

$$
D=\left(d_{1}^{w_{1}} d_{2}^{w_{2}} \ldots \ldots \ldots . . . d_{n}^{w_{n}}\right)^{\frac{1}{\sum w}}
$$

\section{Response Surface Methodology (RSM)}

The response function that represents any of the output features of the weldment can be expressed as 


$$
Y=f(V, W f, T r, N)
$$

Here, $Y$ is the response. $V=$ voltage $(O C V), W f=$ Wire feed rate, $\operatorname{Tr}=$ Traverse Speed and $N=$ electrode stickout.

The selected relationship is a second-degree response surface, which is expressed as follows: -

$$
\begin{aligned}
Y & =\beta_{0}+b_{1} V+b_{2} W f+b_{3} \operatorname{Tr}+b_{4} N+b_{11} V^{2} \\
& +b_{22} W f^{2}+b_{33} \operatorname{Tr}^{2}+b_{44} N^{2} \\
& +b_{12} V . W f+b_{13} V \cdot T r+b_{14} V \cdot N \\
& +b_{23} W f . T r+b_{24} W f . N+b_{34} \operatorname{Tr} . N
\end{aligned}
$$

$$
\beta_{0},\left.b_{i}\right|_{i=1} ^{4},\left.\quad b_{i i}\right|_{i=1} ^{4} \text { and all } b_{i j} \text { (interaction term }
$$
coefficients) are constant. The dimensions (units) of the constants should be such that they must take care of the dimensional similarity on both sides of Eq. (9).

The Response Surface Methodology (RSM) is an efficient tool, which is widely applied for modeling the output response(s) of a process in terms of the important controllable variables and then finding the operating conditions that optimize the response. The Eq. (9) can be written as a multiple linear regression model as follows: -

$$
\begin{aligned}
Y & =\beta_{0}+\beta_{1} x_{1}+\beta_{2} x_{2}+\beta_{3} x_{3}+\beta_{4} x_{4}+\beta_{5} x_{5} \\
& +\beta_{6} x_{6}+\beta_{7} x_{7}+\beta_{8} x_{8}+\beta_{9} x_{9} \\
& +\beta_{10} x_{10}+\beta_{11} x_{11}+\beta_{12} x_{12}+\beta_{13} x_{13}+\beta_{14} x_{14}
\end{aligned}
$$

Here, $Y=f\left(\left.x_{i}\right|_{i=1} ^{14}\right)$ and

$$
\begin{aligned}
\beta_{1} & =b_{1}, \beta_{2}=b_{2}, \beta_{3}=b_{3}, \beta_{4}=b_{4}, \beta_{5} \\
& =b_{11}, \beta_{6}=b_{22}, \beta_{7}=b_{33}, \\
\beta_{8} & =b_{44}, \beta_{9}=b_{12}, \beta_{10}=b_{13}, \beta_{11} \\
& =b_{14}, \beta_{12}=b_{23}, \beta_{13}=b_{24}, \beta_{14}=b_{34} \\
x_{1} & =V, x_{2}=W f, x_{3}=\operatorname{Tr}, x_{4}=N, x_{5} \\
& =V^{2}, x_{6}=W f^{2}, x_{7}=\operatorname{Tr}^{2}, \\
x_{8} & =N^{2}, x_{9}=V . W f, x_{10}=V . T r, x_{11} \\
& =V . N, x_{12}=W f . T r, x_{13}=W f . N, x_{14}=\operatorname{Tr} . N
\end{aligned}
$$

The method of least squares can be used to estimate the regression coefficients in Eq. (10). In this study regression coefficients were computed by statistical software package MINITAB (Release 14).

\section{Particle Swarm Optimization (PSO) Algorithm}

Particle swarm optimization (PSO) is a populationbased stochastic optimization technique developed by Dr. Eberhart and Dr. Kennedy in 1995, inspired by the social behavior of bird-flocking or fish-schooling.
PSO shares many similarities with evolutionary computation techniques such as Genetic Algorithms (GA). The system is initialized with a population of random solutions and searches for optima by updating generations. However, unlike GA, PSO has no evolution operators such as crossover and mutation. In PSO, the potential solutions, called particles, fly through the problem space by following the current optimum particles.

Each particle keeps track of its coordinates in the problem space which is associated with the best solution (fitness) it has achieved so far. (The fitness value is also stored.) This value is called pbest. Another "best" value that is tracked by the particle swarm optimizer is the best value, obtained so far by any particle in the neighbors of the particle. This location is called lbest. When a particle takes all the population as its topological neighbors, the best value is a global best and is called gbest.

The particle swarm optimization concept consists of, at each time step, changing the velocity of (accelerating) each particle toward its pbest and lbest locations (local version of PSO). Acceleration is weighted by a random term, with separate random numbers being generated for acceleration toward pbest and lbest locations.

In the past several years, PSO has been successfully applied in many research and application areas. It has been demonstrated that PSO gets better results in a faster, cheaper way than other methods.

Another reason that PSO is attractive is that there are few parameters to adjust. One version, with slight variations, works well in a wide variety of applications. Particle swarm optimization was used for approaches that can be used across a wide range of applications, as well as for specific applications focused on a specific requirement.

\section{Experimentation}

Bead-on-plate submerged arc welding (on mild steel plates of thickness $10 \mathrm{~mm}$ ) was carried out following 34 full factorial design which consists of 81 combinations of voltage (OCV), wire-feed rate, traverse speed and electrode stick-out. Each process control parameters was varied in three different levels during experiments. Interaction effects of process parameters were assumed negligible in the present study. Three responses related to features of bead geometry viz. bead width, reinforcement and depth of penetration were selected in the present study. A copper coated electrode wire of diameter 3.16 mm (AWS A/S 5.17:EH14) was used during the experiments. Welding was performed with flux (AWS A5.17/SFA 5.17) with grain size 0.2 to $1.6 \mathrm{~mm}$ with basicity index $1.6\left(\mathrm{Al}_{2} \mathrm{O} 3+\mathrm{MnO}_{2} 35 \%, \mathrm{CaO}+\mathrm{MgO} 25 \%\right.$ and $\mathrm{SiO}_{2}+\mathrm{TiO}_{2} 20 \%$ and $\mathrm{CaF}_{2}$ 15\%). The experiments were performed on a Submerged Arc Welding MachineINDARC AUTOWELD MAJOR (Maker: IOL Ltd., India). While the weld was being made, the specimens were prepared for metallographic test. Features of bead geometry (macrostructure) were observed in Optical Trinocular Metallurgical Microscope (Make: Leica, GER- 
Table 1. Process control parameters and their limits

$\begin{array}{lccccc}\text { Parameters } & \text { Units } & \text { Notation } & \text { Level -1 } & \text { Level 0 } & \text { Level +1 } \\ \text { Voltage }(\mathrm{OCV}) & \text { Volts } & \mathrm{V} & 27 & 28 & 29 \\ \text { Wire feed rate } & \mathrm{cm} / \mathrm{min} & \mathrm{Wf} & 655 & 970 & 1285 \\ \text { Traverse speed } & \mathrm{cm} / \mathrm{min} & \mathrm{Tr} & 72 & 98 & 124 \\ \text { Stick-out } & \mathrm{mm} & \mathrm{N} & 27 & 29 & 31\end{array}$

Table 2. Design of experiment and data related to bead geometry parameters

\begin{tabular}{|c|c|c|c|c|c|c|c|}
\hline \multirow[t]{2}{*}{ Sl. No. } & \multicolumn{4}{|c|}{$\begin{array}{l}\text { Design of experiment } \\
\text { (Factorial combination) }\end{array}$} & \multicolumn{3}{|c|}{$\begin{array}{l}\text { Response data } \\
\text { (Bead geometry) }\end{array}$} \\
\hline & $\mathrm{V}$ & Wf & $\operatorname{Tr}$ & $\mathrm{N}$ & $\mathrm{P}$ & $\mathrm{R}$ & W \\
\hline 1 & -1 & -1 & -1 & -1 & 3.849 & 1.761 & 10.061 \\
\hline 2 & -1 & -1 & -1 & 0 & 3.748 & 1.725 & 10.520 \\
\hline 3 & -1 & -1 & -1 & 1 & 3.627 & 1.709 & 11.219 \\
\hline 4 & -1 & -1 & 0 & -1 & 3.472 & 1.374 & 9.151 \\
\hline 5 & -1 & -1 & 0 & 0 & 3.451 & 1.368 & 9.320 \\
\hline 6 & -1 & -1 & 0 & 1 & 3.410 & 1.382 & 9.729 \\
\hline 7 & -1 & -1 & 1 & -1 & 3.155 & 1.287 & 8.821 \\
\hline 8 & -1 & -1 & 1 & 0 & 3.214 & 1.311 & 8.700 \\
\hline 9 & -1 & -1 & 1 & 1 & 3.253 & 1.355 & 8.819 \\
\hline 10 & -1 & 0 & -1 & -1 & 4.149 & 1.836 & 10.980 \\
\hline 11 & -1 & 0 & -1 & 0 & 4.038 & 1.780 & 11.530 \\
\hline 12 & -1 & 0 & -1 & 1 & 3.907 & 1.744 & 12.320 \\
\hline 13 & -1 & 0 & 0 & -1 & 3.762 & 1.446 & 9.720 \\
\hline 14 & -1 & 0 & 0 & 0 & 3.731 & 1.420 & 9.980 \\
\hline 15 & -1 & 0 & 0 & 1 & 3.680 & 1.414 & 10.480 \\
\hline 16 & -1 & 0 & 1 & -1 & 3.435 & 1.356 & 9.040 \\
\hline 17 & -1 & 0 & 1 & 0 & 3.484 & 1.360 & 9.010 \\
\hline 18 & -1 & 0 & 1 & 1 & 3.513 & 1.384 & 9.220 \\
\hline 19 & -1 & 1 & -1 & -1 & 4.649 & 2.067 & 11.559 \\
\hline 20 & -1 & 1 & -1 & 0 & 4.528 & 1.991 & 12.200 \\
\hline 21 & -1 & 1 & -1 & 1 & 4.387 & 1.935 & 13.081 \\
\hline 22 & -1 & 1 & 0 & -1 & 4.252 & 1.674 & 9.949 \\
\hline 23 & -1 & 1 & 0 & 0 & 4.211 & 1.628 & 10.300 \\
\hline 24 & -1 & 1 & 0 & 1 & 4.150 & 1.602 & 10.891 \\
\hline 25 & -1 & 1 & 1 & -1 & 3.915 & 1.581 & 8.919 \\
\hline 26 & -1 & 1 & 1 & 0 & 3.954 & 1.565 & 8.980 \\
\hline 27 & -1 & 1 & 1 & 1 & 3.973 & 1.569 & 9.281 \\
\hline 28 & 0 & -1 & -1 & -1 & 3.638 & 1.565 & 11.671 \\
\hline 29 & 0 & -1 & -1 & 0 & 3.577 & 1.515 & 11.980 \\
\hline 30 & 0 & -1 & -1 & 1 & 3.496 & 1.485 & 12.529 \\
\hline 31 & 0 & -1 & 0 & -1 & 3.321 & 1.208 & 10.121 \\
\hline 32 & 0 & -1 & 0 & 0 & 3.340 & 1.188 & 10.140 \\
\hline 33 & 0 & -1 & 0 & 1 & 3.339 & 1.188 & 10.399 \\
\hline 34 & 0 & -1 & 1 & -1 & 3.064 & 1.151 & 9.151 \\
\hline 35 & 0 & -1 & 1 & 0 & 3.163 & 1.161 & 8.880 \\
\hline 36 & 0 & -1 & 1 & 1 & 3.242 & 1.191 & 8.849 \\
\hline 37 & 0 & 0 & -1 & -1 & 3.888 & 1.670 & 12.550 \\
\hline 38 & 0 & 0 & -1 & 0 & 3.817 & 1.600 & 12.950 \\
\hline 39 & 0 & 0 & -1 & 1 & 3.726 & 1.550 & 13.590 \\
\hline 40 & 0 & 0 & 0 & -1 & 3.561 & 1.310 & 10.650 \\
\hline 41 & 0 & 0 & 0 & 0 & 3.570 & 1.270 & 10.760 \\
\hline 42 & 0 & 0 & 0 & 1 & 3.559 & 1.250 & 11.110 \\
\hline 43 & 0 & 0 & 1 & -1 & 3.294 & 1.250 & 9.330 \\
\hline 44 & 0 & 0 & 1 & 0 & 3.383 & 1.240 & 9.150 \\
\hline 45 & 0 & 0 & 1 & 1 & 3.452 & 1.250 & 9.210 \\
\hline
\end{tabular}


The Journal of Engineering Research Vol. 7, No. 1, (2010) 42-52

\begin{tabular}{|c|c|c|c|c|c|c|c|}
\hline 46 & 0 & 1 & -1 & -1 & 4.338 & 1.931 & 13.089 \\
\hline 47 & 0 & 1 & -1 & 0 & 4.257 & 1.841 & 13.580 \\
\hline 48 & 0 & 1 & -1 & 1 & 4.156 & 1.771 & 14.311 \\
\hline 49 & 0 & 1 & 0 & -1 & 4.001 & 1.568 & 10.839 \\
\hline 50 & 0 & 1 & 0 & 0 & 4.000 & 1.508 & 11.040 \\
\hline 51 & 0 & 1 & 0 & 1 & 3.979 & 1.468 & 11.481 \\
\hline 52 & 0 & 1 & 1 & -1 & 3.724 & 1.505 & 9.169 \\
\hline 53 & 0 & 1 & 1 & 0 & 3.803 & 1.475 & 9.080 \\
\hline 54 & 0 & 1 & 1 & 1 & 3.862 & 1.465 & 9.231 \\
\hline 55 & 1 & -1 & -1 & -1 & 3.523 & 1.509 & 14.101 \\
\hline 56 & 1 & -1 & -1 & 0 & 3.502 & 1.445 & 14.260 \\
\hline 57 & 1 & -1 & -1 & 1 & 3.461 & 1.401 & 14.659 \\
\hline 58 & 1 & -1 & 0 & -1 & 3.266 & 1.182 & 11.911 \\
\hline 59 & 1 & -1 & 0 & 0 & 3.325 & 1.148 & 11.780 \\
\hline 60 & 1 & -1 & 0 & 1 & 3.364 & 1.134 & 11.889 \\
\hline 61 & 1 & -1 & 1 & -1 & 3.069 & 1.155 & 10.301 \\
\hline 62 & 1 & -1 & 1 & 0 & 3.208 & 1.151 & 9.880 \\
\hline 63 & 1 & -1 & 1 & 1 & 3.327 & 1.167 & 9.699 \\
\hline 64 & 1 & 0 & -1 & -1 & 3.723 & 1.644 & 14.940 \\
\hline 65 & 1 & 0 & -1 & 0 & 3.692 & 1.560 & 15.190 \\
\hline 66 & 1 & 0 & -1 & 1 & 3.641 & 1.496 & 15.680 \\
\hline 67 & 1 & 0 & 0 & -1 & 3.456 & 1.314 & 12.400 \\
\hline 68 & 1 & 0 & 0 & 0 & 3.505 & 1.260 & 12.360 \\
\hline 69 & 1 & 0 & 0 & 1 & 3.534 & 1.226 & 12.560 \\
\hline 70 & 1 & 0 & 1 & -1 & 3.249 & 1.284 & 10.440 \\
\hline 71 & 1 & 0 & 1 & 0 & 3.378 & 1.260 & 10.110 \\
\hline 72 & 1 & 0 & 1 & 1 & 3.487 & 1.256 & 10.020 \\
\hline 73 & 1 & 1 & -1 & -1 & 4.123 & 1.935 & 15.439 \\
\hline 74 & 1 & 1 & -1 & 0 & 4.082 & 1.831 & 15.780 \\
\hline 75 & 1 & 1 & -1 & 1 & 4.021 & 1.747 & 16.361 \\
\hline 76 & 1 & 1 & 0 & -1 & 3.846 & 1.602 & 12.549 \\
\hline 77 & 1 & 1 & 0 & 0 & 3.885 & 1.528 & 12.600 \\
\hline 78 & 1 & 1 & 0 & 1 & 3.904 & 1.474 & 12.891 \\
\hline 79 & 1 & 1 & 1 & -1 & 3.629 & 1.569 & 10.239 \\
\hline 80 & 1 & 1 & 1 & 0 & 3.748 & 1.525 & 10.000 \\
\hline 81 & 1 & 1 & 1 & 1 & 3.847 & 1.501 & 10.001 \\
\hline
\end{tabular}

P (Penetration), R (Reinforacement) and W (bead width)

MANY, Model No. DMLM, S6D \& DFC320 and Q win Software). The domain of the experiment is shown in Appendix (Table 1). The design of experiment (DOE) and collected experimental data, related to individual quality indicators of bead geometry are listed in Appendix (Table 2 ). These data were utilized in proposed integrated optimization approach, to be discussed later.

\section{Results and Discussions of Proposed Opti- mization Approach}

\subsection{Calculation of Individual Desirability Values and Overall Desirability Function}

The flow chart of the approach is furnished below in Appendix (Fig. 3). Response data were transformed to their individual desirability values using a desirability function approach (Fuller, D. and Scherer, W., 1998). These are shown in Table 3. For depth of penetration HB
(Higher-the-better) and for reinforcement as well as bead width LB (Lower-the-better) criteria were selected. The index of desirability function was the selected one. In this computation the minimum and maximum values of each response (Table 2) were denoted as $y_{\min }$ and $y_{\max }$ respectively. Individual desirability values of the responses were clustered to calculate the overall desirability value (Table 3). It was assumed that all responses are equally important. The same weight was assigned to all responses.

\subsection{Development of Response Surface Model of Overall Desirability}

RSM was applied to derive a mathematical model of overall desirability. Overall desirability was expressed as a function of four process control parameters. The model consists of linear, square (quadratic) and interaction terms of the process parameters affecting the overall desirability value. The constant term and coefficients of the 
Step 1:

Selection of appropriate desirability function

Step 2:

Transformation of response data into invidual desirability values

\begin{tabular}{|l|l|}
\hline Step 3: \\
\hline Calculation of overall desirability
\end{tabular}

Step 4:

Mathematical modeling of overall desirability using RSM

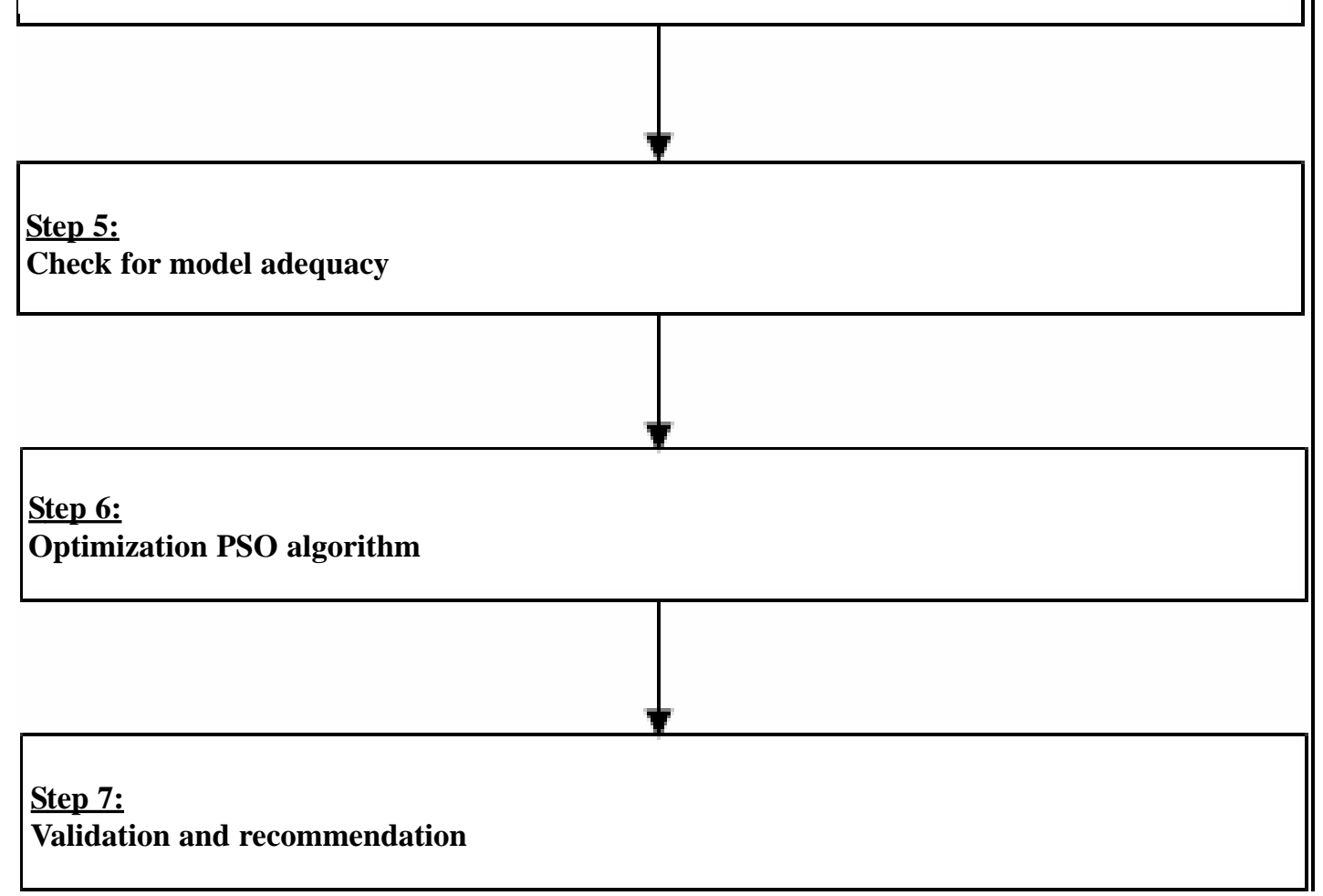

Figure 3. Proposed optimization approach

factors/interaction of factors were evaluated with the statistical-software package Minitab (Release 14). Minitab's multiple linear regression approach was used to derive this model (Eq. 11).

$$
\begin{aligned}
D & =0.625-0.0505 V+0.0317 W f+0.0677 \mathrm{Tr} \\
& +0.0244 N-0.0343 V^{2}-0.0445 W f^{2}-0.112 \mathrm{Tr}^{2} \\
& -0.0315 N^{2}-0.0003 V . W f+0.0166 \mathrm{~V} . \mathrm{Tr} \\
& +0.0049 V . N+0.114 W f . \operatorname{Tr}-0.0131 W f . N \\
& +0.0411 T r . N
\end{aligned}
$$


Table 3. Individual desirability values and calculated overall desirability

\begin{tabular}{|c|c|c|c|c|}
\hline Sl. No. & DP & DR & DW & D \\
\hline 1 & 0.4953 & 0.3280 & 0.8223 & 0.5112 \\
\hline 2 & 0.4315 & 0.3666 & 0.7624 & 0.4941 \\
\hline 3 & 0.3552 & 0.3837 & 0.6712 & 0.4506 \\
\hline 4 & 0.2574 & 0.7428 & 0.9411 & 0.5646 \\
\hline 5 & 0.2442 & 0.7492 & 0.9191 & 0.5519 \\
\hline 6 & 0.2183 & 0.7342 & 0.8657 & 0.5177 \\
\hline 7 & 0.0574 & 0.8360 & 0.9842 & 0.3615 \\
\hline 8 & 0.0946 & 0.8103 & 1.0000 & 0.4248 \\
\hline 9 & 0.1192 & 0.7631 & 0.9845 & 0.4474 \\
\hline 10 & 0.6845 & 0.2476 & 0.7024 & 0.4919 \\
\hline 11 & 0.6145 & 0.3076 & 0.6306 & 0.4921 \\
\hline 12 & 0.5319 & 0.3462 & 0.5275 & 0.4597 \\
\hline 13 & 0.4404 & 0.6656 & 0.8669 & 0.6334 \\
\hline 14 & 0.4208 & 0.6935 & 0.8329 & 0.6241 \\
\hline 15 & 0.3886 & 0.6999 & 0.7677 & 0.5933 \\
\hline 16 & 0.2341 & 0.7621 & 0.9556 & 0.5545 \\
\hline 17 & 0.2650 & 0.7578 & 0.9595 & 0.5776 \\
\hline 18 & 0.2833 & 0.7320 & 0.9321 & 0.5782 \\
\hline 19 & 1.0000 & 0.0000 & 0.6268 & 0.0000 \\
\hline 20 & 0.9237 & 0.0815 & 0.5431 & 0.3444 \\
\hline 21 & 0.8347 & 0.1415 & 0.4281 & 0.3698 \\
\hline 22 & 0.7495 & 0.4212 & 0.8370 & 0.6417 \\
\hline 23 & 0.7237 & 0.4705 & 0.7911 & 0.6458 \\
\hline 24 & 0.6852 & 0.4984 & 0.7140 & 0.6247 \\
\hline 25 & 0.5369 & 0.5209 & 0.9714 & 0.6477 \\
\hline 26 & 0.5615 & 0.5380 & 0.9635 & 0.6627 \\
\hline 27 & 0.5735 & 0.5338 & 0.9242 & 0.6565 \\
\hline 28 & 0.3621 & 0.5380 & 0.6122 & 0.4923 \\
\hline 29 & 0.3237 & 0.5916 & 0.5719 & 0.4784 \\
\hline 30 & 0.2726 & 0.6238 & 0.5002 & 0.4398 \\
\hline 31 & 0.1621 & 0.9207 & 0.8145 & 0.4954 \\
\hline 32 & 0.1741 & 0.9421 & 0.8120 & 0.5107 \\
\hline 33 & 0.1735 & 0.9421 & 0.7782 & 0.5029 \\
\hline 34 & 0.0000 & 0.9818 & 0.9411 & 0.0000 \\
\hline 35 & 0.0625 & 0.9711 & 0.9765 & 0.3898 \\
\hline 36 & 0.1123 & 0.9389 & 0.9806 & 0.4693 \\
\hline 37 & 0.5199 & 0.4255 & 0.4975 & 0.4792 \\
\hline 38 & 0.4751 & 0.5005 & 0.4452 & 0.4731 \\
\hline 39 & 0.4177 & 0.5541 & 0.3617 & 0.4375 \\
\hline 40 & 0.3136 & 0.8114 & 0.7455 & 0.5745 \\
\hline 41 & 0.3192 & 0.8542 & 0.7311 & 0.5842 \\
\hline 42 & 0.3123 & 0.8757 & 0.6854 & 0.5723 \\
\hline 43 & 0.1451 & 0.8757 & 0.9178 & 0.4886 \\
\hline 44 & 0.2013 & 0.8864 & 0.9413 & 0.5517 \\
\hline 45 & 0.2448 & 0.8757 & 0.9334 & 0.5849 \\
\hline 46 & 0.8038 & 0.1458 & 0.4271 & 0.3685 \\
\hline 47 & 0.7527 & 0.2422 & 0.3630 & 0.4045 \\
\hline 48 & 0.6890 & 0.3173 & 0.2676 & 0.3882 \\
\hline 49 & 0.5912 & 0.5348 & 0.7208 & 0.6108 \\
\hline 50 & 0.5905 & 0.5991 & 0.6946 & 0.6264 \\
\hline 51 & 0.5773 & 0.6420 & 0.6370 & 0.6181 \\
\hline 52 & 0.4164 & 0.6024 & 0.9388 & 0.6175 \\
\hline
\end{tabular}


The Journal of Engineering Research Vol. 7, No. 1, (2010) 42-52

$\begin{array}{lllll}53 & 0.4662 & 0.6345 & 0.9504 & 0.6551 \\ 54 & 0.5035 & 0.6452 & 0.9307 & 0.6712 \\ 55 & 0.2896 & 0.5981 & 0.2950 & 0.3711 \\ 56 & 0.2763 & 0.6667 & 0.2742 & 0.3697 \\ 57 & 0.2505 & 0.7138 & 0.2222 & 0.3412 \\ 58 & 0.1274 & 0.9486 & 0.5809 & 0.4126 \\ 59 & 0.1647 & 0.9850 & 0.5980 & 0.4595 \\ 60 & 0.1893 & 1.0000 & 0.5837 & 0.4798 \\ 61 & 0.0032 & 0.9775 & 0.7910 & 0.1346 \\ 62 & 0.0909 & 0.9818 & 0.8460 & 0.4226 \\ 63 & 0.1659 & 0.9646 & 0.8696 & 0.5182 \\ 64 & 0.4158 & 0.4534 & 0.1855 & 0.3270 \\ 65 & 0.3962 & 0.5434 & 0.1529 & 0.3205 \\ 66 & 0.3640 & 0.6120 & 0.0889 & 0.2706 \\ 67 & 0.2473 & 0.8071 & 0.5170 & 0.4691 \\ 68 & 0.2782 & 0.8650 & 0.5223 & 0.5009 \\ 69 & 0.2965 & 0.9014 & 0.4961 & 0.5100 \\ 70 & 0.1167 & 0.8392 & 0.7729 & 0.4230 \\ 71 & 0.1981 & 0.8650 & 0.8160 & 0.5190 \\ 72 & 0.2669 & 0.8692 & 0.8277 & 0.5769 \\ 73 & 0.6681 & 0.1415 & 0.1203 & 0.2249 \\ 74 & 0.6423 & 0.2529 & 0.0758 & 0.2310 \\ 75 & 0.6038 & 0.3430 & 0.0000 & 0.0000 \\ 76 & 0.4934 & 0.4984 & 0.4976 & 0.4964 \\ 77 & 0.5180 & 0.5777 & 0.4909 & 0.5277 \\ 78 & 0.5300 & 0.6356 & 0.4529 & 0.5343 \\ 79 & 0.3565 & 0.5338 & 0.7991 & 0.5337 \\ 80 & 0.4315 & 0.5809 & 0.8303 & 0.5926 \\ 81 & 0.4940 & 0.6066 & 0.8302 & 0.6289\end{array}$

DP (Desirability of penetration), DR (Desirability of reinforcement), DW (Desirability of bead width), OD (Overall desirability)

Table 4. Check for significance of the constant and coefficients in the model

\begin{tabular}{|c|c|c|c|}
\hline Predictor & Coefficient & P-value & Comment \\
\hline Constant & 0.6250 & 0.000 & Significant \\
\hline V & -0.0505 & 0.000 & Significant \\
\hline Wf & 0.0317 & 0.003 & Significant \\
\hline $\operatorname{Tr}$ & 0.0677 & 0.000 & Significant \\
\hline $\mathrm{N}$ & 0.0244 & 0.019 & Significant \\
\hline V2 & -0.0343 & 0.054 & Insignificant \\
\hline $\mathrm{Wf} * \mathrm{Wf}$ & -0.0445 & 0.013 & Significant \\
\hline $\operatorname{Tr} * \operatorname{Tr}$ & -0.1120 & 0.000 & Significant \\
\hline $\mathrm{N} * \mathrm{~N}$ & -0.0315 & 0.076 & Insignificant \\
\hline $\mathrm{V}^{*} \mathrm{Wf}$ & -0.0003 & 0.983 & Insignificant \\
\hline $\mathrm{V}^{*} \operatorname{Tr}$ & 0.0166 & 0.185 & Insignificant \\
\hline $\mathrm{V} * \mathrm{~N}$ & 0.0049 & 0.694 & Insignificant \\
\hline $\mathrm{Wf} * \mathrm{Tr}$ & 0.1140 & 0.000 & Significant \\
\hline Wf*N & -0.0131 & 0.292 & Insignificant \\
\hline Tr*N & 0.0411 & 0.001 & Significant \\
\hline$S=0.0742512$ & $\mathrm{R}-\mathrm{Sq}=78.4 \% \quad \mathrm{R}-\mathrm{Sq}$ & $=73.8 \%$ & \\
\hline
\end{tabular}

The extent of the significance of presence of factors (and interaction of factors) within the model was checked with the Analysis of Variance method (ANOVA) (Table 4). Based on the calculated P-value (probability of significance) of the terms (from Table 4) under considerations, insignificant terms (P-value less than 0.05) were excluded and the final reduced model consisting of significant terms was derived (Eq. 12). This model was optimized (maximized) finally using PSO a algorithm.

$$
\begin{aligned}
D & =0.581-0.0505 V+0.0317 W f+0.0677 \mathrm{Tr} \\
& +0.0244 N-0.0445 W f^{2}-0.112 \mathrm{Tr}^{2} \\
& +0.114 W f . T r+0.0411 T r . N
\end{aligned}
$$




\subsection{Particle Swarm Optimization (PSO)}

In the present study, the reduced mathematical model for overall desirability (Eq. 12) was optimized using a PSO algorithm. It is a constrained optimization problem since the experimental domain was defined by the bounds on the process variables ( $\mathrm{V}, \mathrm{Wf}, \operatorname{Tr}$ and $\mathrm{N})$. The objective is to maximize (Eq. 12) subject to the bounds on the process variables. The values of the parameters of the PSO algorithm used here as follows:

Population size $=50$, Range of Velocity Variation $\mathrm{v}_{\max }$ $=+4, \mathrm{v}_{\min }=-4$, Maximum number of iteration $=100$, Weighting factor $=0.8$, Decrement factor (alpha) $=0.9$ and Social parameters $\mathrm{C} 1=2.0$ and $\mathrm{C} 2=2.0$. By trial and error the values of the aforesaid parameters were chosen so as to improve an objective function value (overall desirability) at the optimal setting.

After optimization the optimal setting becomes:

$$
\left[\begin{array}{c}
V \\
W f \\
T r \\
N
\end{array}\right]=\left[\begin{array}{c}
-0.384 \\
0.916 \\
0.982 \\
0.936
\end{array}\right]
$$

(Optimal value of overall desirability becomes 0.707 ). Figure 4 shows the convergence curve in PSO. Due to non-availability of optimal factors value within equipment's provision, a compromise has to be made. The optimal setting should be modified and set to:

$$
\left[\begin{array}{c}
V \\
W f \\
T r \\
N
\end{array}\right]=\left[\begin{array}{l}
0 \\
1 \\
1 \\
1
\end{array}\right]
$$

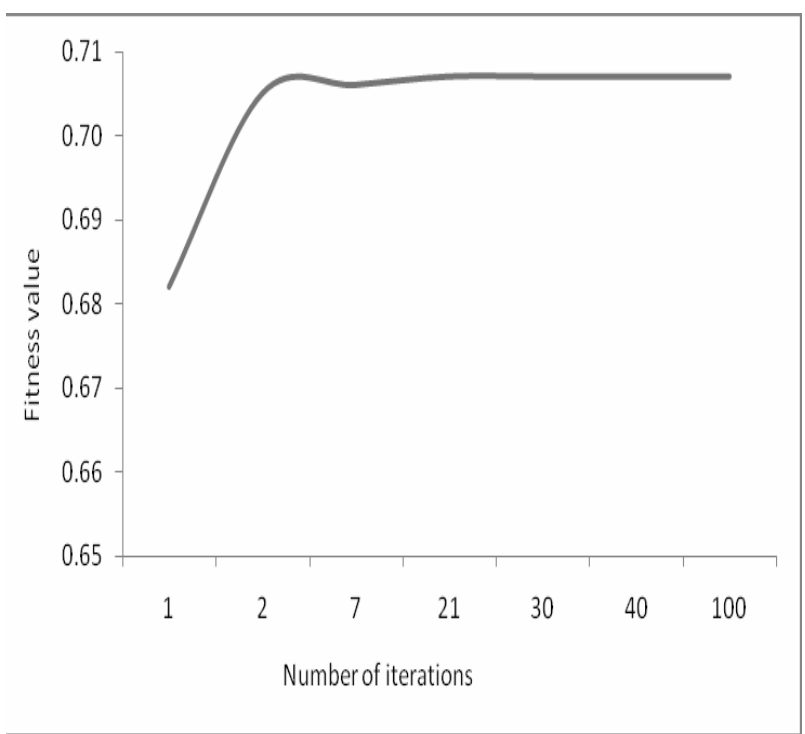

Figure 4. PSO convergence curve

After evaluating the optimal parameter settings, the next step is to predict and verify the enhancement of quality characteristics using the optimal parametric combination. Table 5 reflects the satisfactory result of confirmatory experiment. It indicates that the quality of the weld has improved.
Table 5. Results of confirmatory experiment

\begin{tabular}{ccc} 
& \multicolumn{2}{c}{ Optimal setting } \\
Level of & Prediction & Experiment \\
factors & $V_{0} W f_{1} \operatorname{Tr}_{1} N_{1}$ & $V_{0} W f_{1} \operatorname{Tr}_{1} N_{1}$ \\
Overall & 0.769 & 0.789 \\
desirability & &
\end{tabular}

N.B. Subscripts on factors notation represents factor levels

\section{Conclusions}

Weld quality in SAW depends on features of bead geometry, mechanical-metallurgical characteristics of the weld as well as on weld chemistry. The weld quality improvement is treated as a multi-factor, multi-objective optimization problem. The practical application of SAW requires efficient optimization methodology because process parameters are expected to interact in a complex manner. Therefore, any optimization algorithm must seek to identify interaction effects of input factors and be incorporated in the course of an optimization procedure in a convenient way for developing an efficient methodology. The developed methodology based on RSM, desirability function and PSO algorithm can be applied in practice for continuous quality improvement and off-line quality control. The desirability Function approach converts each of the responses (objectives) into their individual desirability value. Corresponding to each objective, these individual desirability values are then accumulated to compute the overall/composite desirability function, which is to be optimized (maximized) finally. RSM has been applied to derive a mathematical model of overall desirability represented as a function of process control parameters. This mathematical model has been optimized within an experimental domain. Although the paper considers SAW, the procedure is quite generic and can be applied to any process where complex relations among input and output parameters are difficult to predict.

\section{References}

Correia, D.S., Goccalves, C.., Sebastiao, S.C.Junior and Ferraresi, V.S., 2004, " GMAW Welding Optimization using Genetic Algorithms", Journal of Brazilian Society of Mechanical Science and Engineering, 26(1) , DOI: 10.1590/S1678-58782004000100005.

Dongcheol, K., Sehun, R. and Hyunsung, P., 2002, "Modeling and Optimization of a GMA Welding Process by Genetic Algorithm and Response Surface Methodology", International Journal of Production Research, Vol. 40(7), pp. 1699-1711.

Fuller, D. and Scherer, W., 1998, "The Desirability Function: Underlying Assumptions and Application Implications, Systems, Man, and Cybernetics", 1998 IEEE International Conference, Vol. 4, pp. 40164021. 
Hsien-Yu Tseng, 2006, "Welding Parameters Optimization for Economic Design using Neural Approximation and Genetic Algorithm", International Journal of Advanced Manufacturing Technology, Vol. 27(9-10), pp. 897-901.

Leticia, C., Cagnina and Susana, C. Esquivel., 2008, "Solving Engineering Optimization Problems with the Simple Constrained Particle Swarm Optimizer", Informatica, Vol. 32, pp. 319-326.

Price, W.L., 1977, "A Controlled Random Search Procedure for Global Optimization", The Computer Journal, Vol. 20(4), pp. 367-370.

Pasandideh S.H.R. and Niaki, S.T.A., 2006, "MultiResponse Simulation Optimization using Genetic Algorithm within Desirability Function Framework", Applied Mathematics and Computation, Vol. 175(1), pp. 366-382.
Praga-Alejor, R.J., Torres-Trevino, L.M. and PinaMonarrez, M.R., 2008, "Optimization Welding Process Parameters through Response Surface, Neural Network and Genetic Algorithms", Proceedings of the 2008 Electronics, Robotics and Automotive Mechanics Conference, Vol. 00, pp. 393399.

Tay, K.M. and Butler, C., 1996, "Modeling and Optimizing of a MIG Welding Process - a Case Study using Experimental Designs and Neural Networks", Quality and Reliability Engineering International, Vol. 13(2), pp. 61 - 70.

Zhao, X., Zhang, Y. and Chen G., 2006, Model Optimization of Artificial Neural Networks for Performance Predicting in Spot Welding of the Body Galvanized DP Steel Sheets, Advances in Natural Computation, Vol. 4221/2006, DOI 10.1007/11881070. 